\title{
A SEISMIC MODEL OF THE SOLAR CONVECTIVE ENVELOPE
}

\author{
H. SHIBAHASHI ${ }^{1}$, K. M. HIREMATH ${ }^{1,2}$, AND M. TAKATA ${ }^{1}$ \\ ${ }^{1}$ Department of Astronomy, University of Tokyo, Japan \\ ${ }^{2}$ Indian Institute of Astrophysics, Bangalore 560034, India
}

\begin{abstract}
We determine the structure of the solar convective envelope by solving the basic equations for mass conservation and hydrostatic equilibrium with the imposition of the sound-speed profile determined from helioseismology and the equation of state. The solution is required to match with the structure of the radiative core, which is also determined with the imposition of the sound speed profile. The helium abundance is obtained as a part of the solutions.
\end{abstract}

\section{Introduction}

One major success of helioseismology is the determination of the sound speed profile in the solar interior from the observed eigenfrequencies. The inversion has so far been carried out based on either the variational principle or the asymptotic formula. In the former method, where we deal with a small deviation of the true sun from a model, a reference model is always needed. The asymptotic inversion does not necessarily require a reference model, but in order to determine the sound speed profile with a good accuracy, it is better to carry out the linearized or calibrated inversion by introducing a reference model. Usually an evolutionary solar model is used as the reference model. Since the inversion in these cases is a linearized problem, the solution, the deviation of the sound speed profile in the sun from the reference model, becomes more accurate with the introduction of a better reference model. The relative difference between the inverted sound speed profile and the recent evolutionary models is of the order of $1 \%$. If the results from helioseismology can be incorporated in solar modeling, we can improve the solar model and, in turn, we will be able to more accurately determine the sound speed profile of the sun by iteration. Takata and Shibahashi $(1997 \mathrm{a}, \mathrm{b})$ have succeeded in constructing a seismic model of the radiative core of the sun from the observed eigenfrequencies. It is obvious that we also need to construct a seismic model of the convective envelope to compute the theoretical eigenfrequencies of the seismic solar model. Note that the theory of convection is not yet complete. Evolutionary models of the sun are constructed by adjusting the efficiency of the convective energy transport as a free parameter, which is not determined uniquely in the current theory 
of convection. It would be nice if the structure of the solar convective envelope can be helioseismically determined. In this paper, we describe our attempt to construct a convective envelope model from helioseismic data.

\section{Methodology of Making a Seismic Solar Model}

We try to construct a snapshot model of the present-day sun by imposing the soundspeed distribution $c(r)$ obtained from helioseismology (Shibahashi 1993, Shibahashi and Takata 1996a, b, Takata and Shibahashi 1997a, b). We assume that the sun is in hydrostatic equilibrium and in thermal balance. The model is spherically symmetric and we ignore the effects of rotation and the magnetic field. The basic equations for constructing a model with the above assumptions are the same as those used in the theory of stellar structure:

$$
\begin{gathered}
d M_{r} / d r=4 \pi r^{2} \rho, \\
d p / d r=-G M_{r} \rho / r^{2}, \\
d L_{r} / d r=4 \pi r^{2} \rho \varepsilon \\
d T / d r=\left\{\begin{array}{ll}
-\frac{3}{4 a c} \frac{\kappa \rho}{T^{3}} \frac{L_{r}}{4 \pi r^{2}} & \text { if radiative } \\
(d T / d r)_{\text {conv }} & \text { if convective }
\end{array},\right.
\end{gathered}
$$

along with the equation of state and equations for $\kappa$ and $\varepsilon$. Since the sound speed, which we regard as a known function of $r$, is a thermodynamically determined quantity, it is a function of two other thermodynamic quantities, such as $p$ and $T$, along with the mass fraction of each chemical composition $X_{i}$. If we consider hydrogen ${ }^{1} \mathrm{H}$ and helium ${ }^{4} \mathrm{He}$ separately as $X$ and $Y$, respectively, and treat all the other elements collectively as heavy elements $Z$, only two of them should be treated independently because $X+Y+Z=1$. Furthermore, if we know the distribution of $Z(r)$, then only $X$ or $Y$ is regarded as an independent variable; $c=c(p, T, X)$. Inversely,

$$
X=X(p, T, c)
$$

We assume for simplicity that $Z$ is homogeneously distributed. We can then express the density as a function of $c, p$ and $T$ :

$$
\rho=\rho(p, T, X)=\rho(p, T, c) .
$$

Similarly, $Y=Y(p, T, c), \kappa=\kappa(p, T, c)$, and $\varepsilon=\varepsilon(p, T, c)$. Then equations (1)-(4) are a set of equations for $M_{r}, p, L_{r}$, and $T$ for the given $c(r)$ and the given $Z$.

\section{Seismic Model of the Radiative Core}

We divide the solar interior into two parts, the radiative core and the convective envelope, and treat them separately. As for the radiative core, for a given value of $Z$, equations (1)-(4) form a boundary value problem with the following boundary conditions: $M_{r}=0$ and $L_{r}=0$ at $r=0$, and $L_{r}=L_{\odot}$ and $\nabla_{\mathrm{rad}}=\nabla_{\mathrm{ad}}$ at $r=r_{\mathrm{conv}}$ (Takata and Shibahashi 1997a, b). The extent of the convection zone is helioseismologically determined from the kink of $c(r)$ with a reasonable accuracy. We temporarily adopt the thus-determined extent: $r_{\text {conv }} / R_{\odot} \simeq 0.709$. We adopt the OPAL equation of state (Rogers et al. 1996), the OPAL opacity (Iglesias and Rogers 1996), and the 
nuclear reaction rates compiled by Bahcall and Pinsonneault (1995). The profiles of $X(r)$ and $Y(r)$ are obtained as a part of the solutions. The $Z$-value is adjusted so that the ratio $Z / X\left(r_{\text {conv }}\right)$ matches with the spectroscopically obtained value near to the solar surface, since the convection zone is assumed to be chemically homogeneous; $Z / X\left(R_{\odot}\right)=0.0245$ (Grevesse and Noels 1993). The solutions of the radiative core are seen in Takata and Shibahashi (1997a).

\section{Making a Seismic Model of the Convective Envelope}

As for the convective envelope, any physical quantities have already been given at the base of the convection zone from the solution of the radiative core. Since the chemical composition is uniform in the convective envelope and $X$ has been fixed at the base of the convection zone, equation (5) can be regarded as an equation giving $T$ in terms of $p$ and $c(r) ; T=T(p, c)$. Substitution of this into equation (6) allows us to express the density in terms of only $p$ and $c(r) ; \rho=\rho(p, c)$. Then the equations governing the hydrostatic balance (1)-(2) are decoupled from equations (3) and (4). We can determine the density and pressure by solving only equations (1) and (2) by integrating them outward from the base of the convection zone as an initial value problem with the help of equation (6). As for the equations governing the thermal structure, equations (3) and (4), since there is no energy source in the convective envelope, we do not need to consider equation (3). The temperature $T$ is determined from $T=T(p, c)$ and we do not need to solve equation (4), either. In order for the model to be regarded as a solar model, the mass at the solar radius must be equal to the solar mass; $\Delta M=0$, where $\Delta M$ is defined by

$$
\Delta M \equiv M_{r}\left(R_{\odot}\right)-M_{\odot}
$$

and it is dependent on $r_{\text {conv }}, Z / X$, the sound speed profile, and the other microphysics. There is no guarantee that this condition is satisfied. Indeed, for the most likely sound speed profile inverted by Takata and Shibahashi (1997a) from the observational data together with $r_{\text {conv }} / R_{\odot}=0.709$ and $Z / X=0.0245, \Delta M / M_{\odot}$ is $\simeq-2.74 \times 10^{-3}$. Since the uncertainty in the measurement of the mass and the radius of the sun is of the order of $10^{-4},\left|\Delta M / M_{\odot}\right| \simeq 10^{-3}$ is a problem. This discrepancy cannot be compensated by changing $r_{\text {conv }} / R_{\odot}$ or $Z / X$ in their reasonable ranges or by taking account of the uncertainties in the opacity and the nuclear reactions. We have numerically evaluated the dependence of $\Delta M$ upon the uncertainties of the sound speed profile, the depth of the convection zone, and the metal abundance $Z / X$. As a consequence, it was found that $\Delta M$ is highly sensitive to the uncertainty in the sound speed profile, while it is not so sensitive to the uncertainties in the depth of the convection zone and in the metal abundance. Hence we have to adopt some other sound speed profiles rather than the most likely profile. Both the statistical error and the systematic error in the sound speed profile are as small as $0.1 \%$ for $r / R_{\odot}>0.3$, while they are as large as $0.3 \%$ near the center. In order to seek for the sound speed profile with which the seismic model satisfies the boundary condition (7), we modified only the sound speed profile for $r / R_{\odot} \leq 0.35$ by parametrizing how much it deviates at the center from model $\mathrm{S}$ of Christensen-Dalsgaard et al. (1996) (see figure 1), while we kept $r_{\text {conv }} / R_{\odot}=0.709$ and $Z / X=0.0245$. We found that the boundary condition is satisfied for the profile which deviates at the center by $\sim 0.22 \%$ from model $S$. Once we choose this sound speed profile and adopt the above values for $r_{\text {conv }} / R_{\odot}$ and $Z / X$, any physical quantities of both the radiative core and the convective envelope are uniquely determined. The 


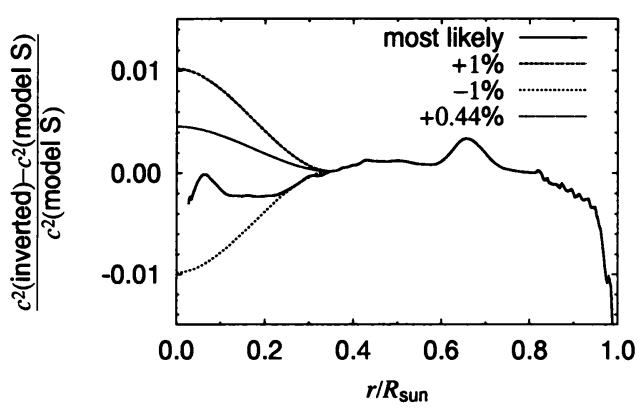

Figure 1. The inverted squared sound speed used as the imposition in solving equations (1)-(4). We modified the most likely profile in $r / R_{\odot}<0.35$, and found that the curve labeled " $+0.44 \%$ " satisfies the outer boundary condition $\Delta M=0$.

resultant chemical abundances are determined as $X=0.755, Y=0.226$, and $Z=$ 0.0185. This is a new method of determining the chemical abundances. The helium abundance has so far been estimated from the local profile of the sound speed at the HeII ionization zone (see e.g., Basu 1997). This method depends on the fact that the function $W(r) \equiv r^{2} /\left(G M_{r}\right) d c^{2} / d r$ has a peak at the He II ionization zone and the peak height is sensitive to the helium abundance. It is then expected that the helium abundance can be well estimated from the sound speed profile at the He II ionization zone. Practically, however, the uncertainty in the equation of state and the quality of the oscillation data produce fairly large errors in the estimation of the helium abundance. On the other hand, the present method of determining the helium abundance is based on the global functional form of $c(r)$. Hence, we think that these two methods should be complementary to each other. We had to modify the sound speed profile within the error bar so that the resultant seismic model satisfies the boundary condition (7). Note that the modification is not unique, and we will have to evaluate more carefully the influence of the error in the sound speed profile upon the seismic model.

\section{References}

Bahcall, J. N. and Pinsonneault, M. H. (1995), Rev. Mod. Phys., 67, pp. 781-808.

Basu, S. (1997), in Proc. IAU Symp. No. 181, Sounding Solar and Stellar Interiors, ed. J. Provost and F.-X. Schmider (Dordrecht: Kluwer), in press.

Christensen-Dalsgaard, J. et al. (1996), Science, 272, pp. 1286-1292.

Grevesse, N. and Noels, A. (1993), in Origin and Evolution of the Elements, ed. N. Prantzos, E. Vangioni-Flam, and M. Cassé (Cambridge: Cambridge Univ. Press), pp. 15-25.

Iglesias, C. A. and Rogers, F. J. (1996), Astrophys. J., 464, pp. 943-953.

Rogers, F. J., Swenson, F. J. and Iglesias, C. A. (1996), Astrophys. J., 456, pp. 902-908.

Shibahashi, H. (1993), in Frontiers of Neutrino Astrophysics, ed. Y. Suzuki and K. Nakamura (Tokyo: Universal Academy Press), pp. 93-103.

Shibahashi, H. and Takata, M. (1996a), Publ. Astron. Soc. Japan, 48, pp. 377-387.

Shibahashi, H. and Takata, M. (1996b), Bull. Astron. Soc. India, 24, pp. 301-304.

Takata, M. and Shibahashi, H. (1997a), Astrophys. J., submitted.

Takata, M. and Shibahashi, H. (1997b), in these proceedings. 